\title{
Contratos de integración vertical en la producción agraria: Posicionamiento del orden jurídico argentino
}

Contracts of vertical integration in agricultural production: Positioning of the Argentine legal order

Contratos de integração vertical na produção agrária: o posicionamento do ordenamento jurídico argentino

Les contrats d'intégration verticale dans la production agraire: positionnement de l'ordre juridique argentin

Noelia García ${ }^{1}$ | Universidad Nacional de La Plata

Revista Derechos en Acción

Año 3/NNo 9 Primavera 2018, 357-372

DOl: https://doi.org/10.24215/25251678e225

ORCID: https://orcid.org/0000-0002-1977-4220

Recibido: 11/09/2018

Aprobado: 17/11/2018

Resumen. Este trabajo se propone brindar una aproximación a las formas en que se estructuran las cadenas agroindustriales, detallando las ventajas y desventajas relativas que presentan para sus distintos eslabones. Asimismo, analizará las implicancias económicas de las diversas variantes de integración, las formas características que adopta la producción a nivel nacional, cómo esta nos posiciona en el mercado actualmente y cuál es el rol que se pretende desempeñar a futuro; esto último, a fin de evaluar si el ordenamiento jurídico acompaña o no dicho plan.

\footnotetext{
Abogada graduada en la UNLP. Integrante de la Clínica Jurídica de Derechos Humanos de la Facultad de Ciencias Jurídicas y Sociales de dicha casa de estudios. Desempeño funciones en la Contaduría General de la Provincia. Contacto: noegarpr@gmail.com.
} 
Palabras clave: integración vertical; agroalimentario; agroindustrial; valor agregado.

Abstract. The aim of this paper is to present the ways that agroindustrial chains are currently organized, including the advantages and disadvantages that such chains bring about for each of the existing links. We will also analyse the economic consequences of the different forms of integration, the typical forms production may take on a national scale, the consequent position within the market and the goals to be achieved in the future. All said factors shall be considered with the purpose of assessing the adequacy of the Argentina's legal system to fulfill the above mentioned goals.

Keywords: vertical integration; agri-food; agro-industrial; value added $\operatorname{tax}$ (VAT).

Resumo. 0 objetivo deste artigo é fornecer uma aproximação ás formas pelas quais as cadeias agroindustriais são estruturadas, detalhando as vantagens e desvantagens relativas que elas apresentam para seus diferentes passos. Da mesma forma, analisará as implicações econômicas das diferentes variantes de integração, as formas características que adota a produção a nível nacional, como isso nos posiciona no mercado atualmente e qual é o papel que pretende desempenhar no futuro; 0 último, a fim de avaliar se o sistema jurídico acompanha ou não o referido plano.

Palavras-chave: integração vertical; agroalimentario; agroindustrial; valor adicionado.

Résumé: Ce travail se propose d'apporter une approximation aux manières dans lesquelles les chaines agroindustrielles forment leurs structures, en détaillant les avantages et inconvénients relatifs qui ses chaînons présentent. Aussi, on analysera les conséquences économiques des diverses variantes d'intégration, les formes caractéristiques qui la production à niveau mondial adopte, la manière dans laquelle il nous positionne dans le marché actuel, et quel est son rôle futur; afin d'évaluer si l'ordre juridique accompagne ce plan.

Mot-clés: intégration verticale, agroalimentaire, agroindustriel, valeur ajoutée 


\section{Introducción}

El escenario económico actual se presenta como una oportunidad propicia para replantearnos -entre otras cosas- el rol que desempeña la Argentina en el mercado mundial como máxime exportador de commodities. En efecto, la devaluación de la moneda nacional con el correlativo ascenso del dólar nos invitan a repensar una vez más qué tan vetusto es sostener en el presente siglo el modelo agroexportador: con él, nos ubicamos en el primer eslabón de una cadena de valor que se lleva a cabo fronteras afuera y de cuyos beneficios nos excluimos, obnubilados por las divisas que nos dejan las exportaciones de materias primas.

No se pretende aquí brindar una solución acabada a tan complejos problemas pero sí delinear las relaciones jurídicoeconómicas que dan lugar a las cadenas agroindustriales; ello, para entender las implicancias del rol que ocupan nuestros productores y el costo de oportunidad que tal posición conlleva. Finalmente, este trabajo exploratorio permitirá advertir la escasa regulación jurídica en la materia invitándonos a pensar qué herramientas puede brindar el derecho a una política alternativa a aquel modelo.

\section{El asociativismo agrario. Marco conceptual}

Para poder adentrarnos en el tema que nos convoca es necesario describir el contexto en el cual la integración tiene lugar, las diferentes circunstancias que conllevan a la misma (dadas las ventajas que implica) como así también sus diversos tipos.

El proceso de industrialización, la progresiva mejora en los medios de transporte y comunicaciones -con el consecuente proceso de globalización- y el hecho de que naturalmente la producción agraria dé lugar a un mercado de commodities altamente competitivo, han constituido las condiciones propicias para generar distintas formas de asociativismo agrario. Se entiende por tal al conjunto de estrategias tendientes a agrupar esfuerzos 
entre productores o empresas agrarias y tutelarse mutuamente (cfr. Pastorino, 2009, pág. 523).

Es en ese marco que podemos hablar de integración empresarial, conceptualizándola -de manera muy rudimentaria, por ahora- como el conjunto de relaciones que vinculan y reúnen a los distintos eslabones de una cadena productiva. Primariamente, podemos distinguir entre integración horizontal y vertical; la primera de ellas tiene lugar cuando se vinculan empresas o productores agrarios para realizar conjuntamente una misma actividad en su integridad (integración horizontal total) o al menos, para concurrir en determinados aspectos de la misma (integración horizontal parcial). Un ejemplo de lo primero se da cuando varios productores adquieren en común vacas lecheras, invierten conjuntamente en la instalación del tambo y comercializan de la misma manera la leche obtenida; lo segundo se daría si se reúnen al sólo efecto de comercializar la leche juntos y lograr de esa manera una posición más competitiva (obtener un mejor precio) frente al industrial. ${ }^{2}$ Esta forma de integración otorga como ventaja, además de la ya referida competitividad, la reducción de costos -en tanto facilita emprendimientos que individualmente pueden ser muy onerosos y difíciles de afrontary la distribución de riesgos.

La integración vertical, en cambio, implica el establecimiento de relaciones más o menos estrechas y estables entre los distintos eslabones que componen una cadena productiva. Decimos "más o menos estrechas" porque, nuevamente, podemos distinguir entre integración vertical total (o por propiedad) o parcial: la primera tendrá lugar cuando toda la cadena productiva obedece a una unidad de mando y gestión constituyendo, en el más avanzado de los casos, una única empresa; comparado con el ejemplo anterior, sería el caso de una industria láctea que reúne en sí misma -bajo una única firma- las facetas de producción de materia prima, transporte, industrialización, comercialización y distribución.

2 El cooperativismo suele ser con frecuencia una de las formas que toma la integración horizontal. 
Aunque no se lo advierta a simple vista, las economías de subsistencia se caracterizaron por la integración total: históricamente, la familia era creadora de sus alimentos, concentrándose entonces la producción y el consumo en una misma unidad.

Como contrapartida, la integración vertical parcial (o por contratos coordinados) se configura cuando los diversos eslabones de la cadena mantienen su autonomía (jurídica, al menos) pero se relacionan de manera estable entre sí a través de contratos que tienen como notas características las siguientes: a) se prevén pautas ineludibles en cuanto a insumos a utilizar, calidad de la producción, cantidad, tiempo de entrega o disponibilidad etc.; y b) se dispone algún mecanismo de control por parte de la empresa que ocupa un lugar de poder en la cadena -normalmente el industrial- respecto de los demás integrantes de la misma, dando la nota de "verticalidad" . Analizando en detalle el supuesto, podemos realizar una nueva diferenciación y hablar de integración hacia atrás (o descendente) y hacia adelante (o ascendente) según la etapa productiva que proponga la integración: en el primer caso, una empresa proyecta asumir o incorporar etapas anteriores a su actividad y hasta ese momento ajenas a la misma (como sería la integración de un proveedor), mientras que en el segundo lo que se incorpora es una actividad ulterior -de la cadena de valor- a la actualmente realizada (como sería la integración de un distribuidor o comercializador). Empleamos la expresión "incorporar" porque, efectivamente, quien propone la integración es una empresa consolidada en la actividad y con suficiente poder económico y organizacional como para controlar y dirigir fases de la producción que le eran ajenas hasta ese momento: por eso se habla también de integrador e integrados a una cadena.

Aquí la situación es más compleja porque requiere la existencia de múltiples contratos que, precisamente, encadenen las

3 El pacto de exclusividad es muy común en estos tipos de integración. Por ejemplo, el productor se compromete a que el industrial del acuerdo sea el único comprador de su mercancía. 
distintas fases de la actividad, por lo cual las características y el grado de integración dependerá de cada caso concreto y variará para cada unión de eslabones: las condiciones que se pactan entre el productor y el industrial difieren de las que caracterizan la vinculación entre este último y el distribuidor, siendo también relativo el grado de poder de cada uno de ellos.

Este último tipo de integración brinda previsibilidad en las relaciones económicas, permitiendo establecer de antemano la demanda que tendrán las materias primas y/o productos elaborados como asimismo su precio aproximado. Es frecuente en estos supuestos que el productor tenga vendida la materia prima antes de su obtención, lo que otorga seguridad en el desarrollo de la actividad.

A su vez -y a diferencia de lo que sucede en la integración vertical total, como se verá infra- los riesgos característicos de cada etapa productiva son asumidos por cada una de ellas, sin extenderse de manera directa a las restantes. Sin embargo, decimos que las unidades mantienen "al menos" su autonomía jurídica porque la integración vertical parcial suele quitar autonomía económica, generando -la más de las veces- una gran dependencia: sobre todo para los integrados respecto del integrador.

Claro que la integración vertical total brinda no sólo previsibilidad sino, más bien, total control sobre todas las fases de la producción (al reunirse ésta bajo un único mando o, incluso, propiedad). Pero, del mismo modo, ello implica asumir en una única gestión la totalidad de los riesgos que cualquier actividad económica trae aparejada, situación que puede predicarse también respecto de la responsabilidad jurídica. Los riesgos se potencian en este caso al repercutir en la misma unidad administrativa-organizacional y patrimonial las fluctuaciones que alcancen a cualquier eslabón o fase, como serían los caso de aumento de costos de los insumos en la fase productiva o aumento de combustible (por nombrar casos que de modo patente, en condiciones de integración parcial, afectarían principalmente a los productores primarios y transportistas $\mathrm{o}$ 
distribuidores, respectivamente $)^{4}$. Otras desventajas radican en la dificultad que se presenta para externalizar costos fijos y la pérdida de flexibilidad para volcarse a otras actividades y/o mercados.

\section{Implicancias económicas}

Mencionamos ya que la integración vertical da lugar, en los hechos, a relaciones de poder y dependencia económica de una faz productiva respecto de otra; dependencia que obedecerá al tipo de actividad y a los términos de cada contrato asociativo en particular. Sin embargo, para analizar dichos supuestos podemos acudir a dos conceptos que nos permitirán evaluar la calificación o el posicionamiento relativo de una empresa en la cadena industrial: amplitud y extensión. En tal sentido, la amplitud de una integración indica el grado en que una empresa depende de sus propios recursos internos para satisfacer sus necesidades de insumos o para comercializar sus productos, mientras que la extensión se refiere a la longitud de la cadena de valor que posee una empresa (cfr. Victoria, 2012, pág. 83-84).

Entonces, para medir uno y otro concepto nos podemos valer de dos herramientas claves: dependencia a insumos externos, ajenos a la unidad productiva de que se trate (en el primer caso) y fracción de valor agregado por la unidad productiva o empresa en el valor final del producto al transitar toda la cadena (en el segundo supuesto).

Así -y siguiendo siempre el mismo ejemplo- un tambero ya consolidado en su actividad cuenta con la mayor parte de los insumos que requiere para llevar a cabo la misma; situación que sobresale aún más si se lo compara con el eslabón siguiente -el industrial- que para poder generar productos lácteos requiere necesariamente de materia prima (leche) ajena a su empresa.

4 Téngase en cuenta que en el supuesto de integración vertical parcial los precios suelen pactarse anticipadamente, por lo que la posibilidad de trasladar costos al interior de la cadena se ve notoriamente reducida. 
Desde esta perspectiva, podría decirse que el tambero es más independiente y tiene una mejor posición que el industrial. Sin embargo, ni bien se analiza el caso a través del otro concepto (la extensión) resulta fácil advertir que mientras el tambero comercializa el fruto de su actividad sin agregarle valor alguno -o siendo este muy escaso- el industrial es quien aporta la fracción más relevante de valor agregado en el producto final de la cadena, situación que lo coloca con mayor poder relativo frente a las fases restantes: “...de toda la cadena productiva-comercial, solamente el 15 al 25\% del valor final del producto puesto en la góndola lo recibe el productor primario. La principal ganancia la captan los intermediarios, los comercializadores y los expendedores (góndolas)" (INTA, 2011, pág. 1).

Con estas herramientas podemos advertir que, en el caso analizado, el industrial tiene una fuerte dependencia del productor y su suerte está atada, en cierta medida, a la de aquel. Sin embargo, el hecho de aportar valor agregado le da al primero de ellos grandes ganancias y posicionamiento en el mercado lo que -sumado a la dispersión de los productores y su consecuente debilidad en términos relativos- le otorga fuerza de negociación, control e imposición de reglas, notas típicas como ya vimos, de la verticalidad.

Es por ello que en la práctica, las formas de integración (vertical y horizontal, con sus variantes) no aparecen de manera pura sino que suelen combinarse: tamberos que se unen entre sí a través de cooperativas ${ }^{5}$ para adquirir peso en la comercialización; industriales que establecen relaciones estrechas con dichas cooperativas exigiéndoles cierta calidad, el empleo de determinados insumos, pactando compras a precios ya determinados, reservándose facultades de control, etc. ${ }^{6}$ Tampoco se

5 También se acude a los consorcios y asociaciones civiles como formas de organizar la integración horizontal.

6 En el derecho comparado se desarrollan acuerdos de integración en su modalidad colectiva: son acuerdos interprofesionales que vinculan agrupaciones representativas de los agricultores, por un lado, y de los comerciantes, por el otro. Estos acuerdos constituirían el 
puede dejar de reseñar en esta instancia que los eslabones que conforman estas cadenas agroindustriales trascienden las fronteras nacionales, dato que es indispensable ni bien se analiza la producción nacional.

En nuestro caso, predominan las actividades con bajo valor agregado (promedio de U\$S 700 por tonelada exportada), mientras que hay una fuerte prospección a importar con alto valor agregado (U\$S 1.800 la tonelada importada), diferencia -entre el valor de la tonelada exportada e importada- que genera un déficit de 400.000 puestos de trabajo y alta dependencia de manufacturas de origen industrial: $90 \%$ del total importado, frente al $30 \%$ del total exportado (cfr. INTA, 2011, pág. 3).

Por lo tanto, en términos de extensión no podemos más que afirmar que los productores locales tienen una escasa participación en el valor agregado a lo largo de todo el ciclo industrial, lo que -además del desequilibrio en la balanza de pagos que esto conlleva- implica pérdidas comparativas en materia fiscal: si a largo plazo se diseñaran políticas tendientes a aumentar el valor agregado de origen nacional, fomentando la participación en términos competitivos de los productores -asociación horizontal mediante- en la industrialización y/o transformación de las materias primas en alimentos y/o biocombustibles, además de generarse numerosas fuentes de trabajo se incrementaría significativamente la recaudación impositiva a nivel interno; basta a tal fin con pensar solo en el IVA. Estos han sido los objetivos explicitados en el Plan Estratégico Agroalimentario y Agroindustrial Participativo y Federal 2010-2020 (PEA):

El desafío de este período, y que deberá plasmarse en el PEA, será cómo aprovechar adecuadamente las oportunidades de un mundo emergente en rápido crecimiento, balanceando debidamente el mejor rédito a obtener del agro, teniendo en cuenta los factores económicos,

marco de cláusulas mínimas e imperativas (junto a otras de carácter supletorio) a las cuales estarían subordinados los contratos individuales que conformen luego cada integración en concreto (cfr. Pastorino, 2009, pág. 530). 
regionales, ambientales y de empleo, sabiendo además, que la asignatura pendiente es lograr un mayor agregado de valor a la producción primaria y de primera industrialización (Presidencia de la Nación, 2010, pág. 72).

Allí se proyectan y estiman comparativamente los resultados de dos modelos alternativos de producción: el incremental y el de valor agregado con desarrollo. El primero es el que la Argentina conoce hasta ahora: ser generadora de commodities exportables buscando de manera constante tan sólo el incremento en su producción; el segundo propone -en cambiogenerar valor agregado en origen, no solo tendiendo a insertar con mayor peso al país en las cadenas globales de valor sino, y ante todo, procurando que haya cadenas íntegras a nivel nacional que den lugar a exportaciones de bienes y servicios finales. En virtud del crecimiento exponencial que arrojaría el segundo modelo por sobre el primero, se diseña finalmente el plan estratégico para alcanzarlo, el que se compone de diferentes fines y objetivos (cfr. Presidencia de la Nación, 2010, cap. 5).

Excede el marco de este trabajo profundizar en cada una de las metas propuestas y los medios elegidos para conseguirlas. Lo que incumbe destacar es el inmenso interés político-económico que revisten las cadenas agroindustriales y qué tan determinante resulta la posibilidad de cambiar el posicionamiento de los productores locales en términos de extensión, decisión esta última que ya ha sido ponderada por las autoridades con resultados obvios: hay que lograr para nuestros productores la mayor extensión posible en la integración.

Pues bien, ¿cuál es el rol que le cabe al derecho en este proyecto? Dentro de los fines estratégicos-institucionales se persigue "promover la legislación necesaria para el pleno y sustentable desenvolvimiento de las actividades agroalimentarias y agroindustriales" e "implementar un régimen fiscal, comercial y de competencia equitativo desde lo social y alentador para la inversión privada" (Presidencia de la Nación, 2010, pág. 162). Veamos entonces cuál es el estado actual del orden jurídico argentino en la materia. 


\section{III. ¿Vacío legal?}

Forzoso es comenzar este estudio examinando los términos de la ley $\mathrm{N}^{\circ} 25.113$, regulatoria del contrato de maquila ${ }^{7}$ y de los contratos agroindustriales.

Según esta, el primero se configura cuando el productor agropecuario se obliga a suministrar al procesador o industrial materia prima con el derecho de participar, en las proporciones que convengan, sobre el o los productos finales resultantes, los que deberán ser de idénticas calidades a los que el industrial o procesador retengan para sí. Se destaca también como rasgo típico que el productor agropecuario mantiene durante todo el proceso de transformación la propiedad sobre la materia prima y luego sobre la porción de producto final que le corresponde (Ley 25.113,1999, art. 1).

No obstante las bondades que presenta la norma, colocando las facultades de control en cabeza del productor y prohibiendo el pacto de exclusividad (Ley 25.113,1999, arts. 2 inc. e, 3 y 4), es difícil imaginar el empleo de esta figura en materia de integración vertical: justamente porque estas limitaciones -aunque loables y protectorias- van notoriamente en contra de las características reseñadas ut supra para este tipo de integración. A mayor abundamiento, nótese que sólo hay contrato de maquila cuando la participación o contraprestación del productor es en especie del resultado final obtenido, lo que nuevamente dificulta su aplicación a las grandes cadenas agroalimentarias.

Es aquí donde se cuela la figura de los contratos agroindustriales, es decir, aquellos que tienen por objeto la provisión de materia prima de naturaleza agropecuaria para su procesamiento, industrialización y/o transformación (Ley 25.113,1999, art. 6) ${ }^{8}$. Como puede observarse, si bien ya no se exige el requisito de la

\footnotetext{
7 No entraremos aquí en el debate relativo a si el contrato de maquila es o no una especie de contrato agroindustrial.

8 En virtud del referido artículo, las disposiciones de la Ley relativas al contrato de maquila se aplican también a los contratos agroindustriales.
} 
participación sobre el producto final siguen quedando afuera las facetas de comercialización y distribución, en virtud de lo cual sólo de manera parcial y muy rudimentaria podría sostenerse que se subsumen en esta figura los contratos configurativos de las cadenas agroindustriales (cfr. Pastorino, 2009, pág. 529).

En síntesis, la ley $N^{\circ} 25.113$ no conforma un régimen acabado y contemplativo de las todas relaciones que configuran una integración vertical pudiendo -en el mejor de los casos- aplicarse tan sólo al contrato que vincula al productor con el industrial, supuesto en el cual sí resultan meritorias sus disposiciones al erigirse en un orden protectorio del primero de ellos.

Superado el análisis de dicha normativa, resta indagar en la integridad del sistema jurídico de manera que del conjunto de sus disposiciones puedan extraerse principios rectores, directrices capaces de orientar la toma de decisiones -ya sea judiciales o de futuras reformas legislativas- en la materia.

En tal sentido, la Constitución Nacional pone a cargo de las autoridades el deber de proveer a la defensa de la competencia contra toda forma de distorsión de los mercados (Const., 1994, art. 41), mientras que el Código Civil y Comercial -haciéndose eco de aquella y en el marco de la comunicabilidad entre el derecho público y privado (cfr. Lorenzetti, 2014, pág. 66)- hace aplicable el principio de buena fe y la doctrina del abuso del derecho a quien se abuse de una posición dominante en el mercado, "sin perjuicio de las disposiciones específicas contenidas en leyes especiales" (Código civil y comercial, 2014, art. 11).

Puede observarse que el Código no conceptualiza el término "posición dominante del mercado" y ello se debe a una única razón: en el marco de la comunicabilidad antes reseñada, tal definición sigue en manos del derecho público; específicamente, serán las previsiones de la ley de defensa de la competencia las que determinarán cuándo se configura el supuesto, respecto del cual el Código agrega tan sólo algunos efectos jurídicos: la aplicación de los principio rectores de la buena fe y el abuso del derecho (Código civil y comercial, 2014, arts. 9 y 10) y la 
posibilidad de accionar de manera preventiva y resarcitoria según las disposiciones de este último (cfr. Lorenzetti, 2014, pág. 65).

A su turno, la ley $\mathrm{N}^{\circ} 25.156$ de defensa de la competencia se encarga del tema de manera muy precisa:

(...) se entiende que una o más personas goza de posición dominante cuando para un determinado tipo de producto o servicio es la única oferente o demandante dentro del mercado nacional o en una o varias partes del mundo o, cuando sin ser única, no está expuesta a una competencia sustancial o, cuando por el grado de integración vertical u horizontal está en condiciones de determinar la viabilidad económica de un competidor participante en el mercado, en perjuicio de éstos (Ley 25.156, 1999, art. 4).

Encontramos aquí la única referencia concreta a la integración en sus distintas variantes -vertical u horizontal- como una de las modalidades que puede adoptar la posición dominante del mercado: si es capaz de determinar la viabilidad económica de la competencia. Para delimitar este último supuesto puede servirnos de ayuda lo dispuesto en el art. 5 inc. c): evaluar si la integración es capaz (y en su caso, en qué medida) de influir unilateralmente en la formación de precios o restringir el abastecimiento o demanda del mercado y el grado en que sus competidores puedan contrarrestar dicho poder (Ley 25.156, 1999, art. 5 inc. c).

En definitiva y hasta el momento, si bien el ordenamiento advierte la existencia de estas cadenas agroindustriales la regulación es ínfima y abarca tan sólo una de las aristas posibles: la distorsión de los mercados a que las mismas pueden, en ciertas condiciones, arribar. Nuevamente, esta es una finalidad estimable y que no debe abandonarse pero, como ya lo anticipara el PEA, no debería ser la única a tener en cuenta por el legislador.

Es que la realidad nos enseña que estas cadenas, con los múltiples y variados vínculos a que dan lugar, no sólo existen (independientemente de que cada eslabón mantenga su autonomía y personalidad jurídica) sino que desempeñan un rol fundamental 
en la producción, participación que es necesario reforzar procurando mayor valor agregado a nivel nacional. Repensar el derecho en estos términos seguramente contribuirá a tales fines.

Como una tímida muestra de que dichos vínculos no sólo son advertidos sino que cobran relevancia jurídica, cabe mencionar tres supuestos a nivel normativo: I) la ley de defensa del consumidor, que responsabiliza de manera solidaria a todos los integrantes de la cadena por los daños que aquel sufra en virtud del riesgo o vicio de la cosa, con total prescindencia del grado y el tipo de integración que los une (Ley 24.240, 1993, art. 40); II) la ley de contrato de trabajo, que atribuye la misma responsabilidad a las empresas que -aunque tengan personalidad jurídica propia- sean coordinadas, dirigidas o administradas por otras, o estuvieran de tal modo relacionadas que constituyan un conjunto económico de carácter permanente, cuando hayan mediado maniobras fraudulentas o conducción temeraria ${ }^{9}$ (Ley 20.744, 1976, art. 31); y III) el Código Civil y Comercial que entiende por conexos a aquellos contratos vinculados entre sí por una finalidad económica común, de modo que uno es determinante del otro para el logro del resultado perseguido. Dicha finalidad puede derivarse de la interpretación: los contratos conexos se explican unos por medio de los otros y adquieren sentido como un todo sistémico, según su función económica y el resultado perseguido. Como consecuencia de ello, un contratante puede oponer las excepciones de incumplimiento aún frente a la inejecución de obligaciones ajenas a su contrato, permitiéndose asimismo alegar la imposibilidad de cumplimiento cuando la extinción de un contrato conexo produce la frustración de la finalidad económica común (Código civil y comercial, 2014, arts. 1073, 1074 y 1075).

Si el principio de primacía de la realidad nos permite en materia laboral prescindir de la delimitación que implica la existencia de distintas personas jurídicas para ver -conforme los lazos

9 Siendo todas ellas solidariamente responsables respecto de las obligaciones asumidas con sus trabajadores y ante los organismos de la seguridad social. 
permanentes que las unen- un conjunto económico; si la ley de defensa del consumidor hace lo propio en materia de responsabilidad por daños; y si -en la misma dirección- el Código Civil y Comercial permite usar defensas en una relación contractual distinta a la que le sirve de causa en virtud de la conexidad ínsita a la finalidad económica común, quizás sea hora de que el legislador se aggiorne en materia de integración económica y refleje en el derecho la realidad imperante: no con el único afán de regular la institución, sino para repensar el papel de los productores nacionales y fomentar el agregado de valor a nivel local. En definitiva, para aproximarnos a las metas del PEA.

Una buena política necesita de reglas congruentes con sus objetivos, que generen los incentivos adecuados para su persecución.

\section{Conclusión}

Como se habrá advertido, la idea en torno a la que gira este trabajo y en virtud de la cual se insta a una regulación adecuada de la integración agroalimentaria -principalmente en su modalidad vertical- es la dependencia en que se encuentra el productor frente al industrial. Eslabón este último que en el caso argentino y dado el gran peso que representan las materias primas en nuestras exportaciones, suele operar desde el exterior.

La creciente independencia de los productores nacionales que traería aparejada una integración vertical ascendente, donde estos incorporen las facetas posteriores -que son justamente las que mayor valor agregan a la cadena- se traduciría en un considerable incremento de divisas, aumento en la recaudación interna y crecimiento de las fuentes de trabajo, expresándose correlativamente en términos de mayor soberanía nacional.

\section{Referencias bibliográficas}

BRAGACHINI, M., CASINI, C., SAAVEDRA, A., MÉNDEZ, J., ERRASQUIN, L., USTARROZ, F. y BRAGACHINI, M., (2011). Argentina 
hacia la industrialización del campo Argentino 2011/2020. Boletín del Proyecto Nacional de Eficiencia de Cosecha, Poscosecha y agregado de Valor en Origen - INTA PRECOP III. Recuperado de https://inta.gob.ar/sites/default/files/scripttmp-argentina_hacia_la_industrializacin_del_campo.pdf

LORENZETTI, R. L. (2014) Código Civil y Comercial de la Nación Comentado. Santa Fe, Argentina: Rubinzal- Culzoni.

PASTORINO, L. (2009). Derecho agrario argentino. Buenos Aires, Argentina: Abeledo Perrot.

PRESIDENCIA DE LA NACIÓN, (2010). Plan Estratégico Agroalimentario y Agroindustrial Participativo y Federal 20102020. Recuperado de https://inta.gob.ar/sites/default/files/ inta_000001-libro_pea_argentina_lider_agroalimentario.pdf

VICTORIA, M. A. (2012). Integración vertical para los agronegocios. Revista Académica CIFRA 7, de la Facultad de Humanidades, Ciencias Sociales y de la Salud de la Universidad Nacional de Santiago del Estero, ISSN: 0328-8862, pp. 77-96 Recuperado de http://fhu.unse.edu.ar/carreras/rcifra/victoria.pdf.

\section{Legislación consultada}

Código Civil y Comercial [Código]. (2014) 1ra ed. La Ley.

Congreso de la Nación Argentina (13 de Mayo de 1976) Régimen de Contrato de Trabajo [Ley 20.744 de 1976].

Congreso de la Nación Argentina (22 de Septiembre de 1993) Defensa del Consumidor [Ley 24.240 de 1993].

Congreso de la Nación Argentina (23 de Junio de 1999) Contratos de Maquila [Ley 25.113 de 1999].

Congreso de la Nación Argentina (25 de Agosto de 1999) Defensa de la Competencia [Ley 25.156 de 1999].

Constitución de la Nación Argentina [Const.] (1994) 1ra Ed. Producciones Mawis. 